\title{
O movimento DIY na década de 1970 e a difusão de um mobiliário nômade: Nomadic Furniture e How to build your own living structures
}

The DIY movement in the 1970s and the diffusion of a nomadic furniture: Nomadic Furniture and How to build your own living structures

OLIVEIRA, Amanda Aline Alves; Mestranda em Design e Arquitetura; FAUUSP

amandaaline212@usp.br

SAKURAI, Tatiana; Doutora; FAUUSP

tsakurai@usp.br

\section{Resumo}

Seria a década de 1970 com todos os seus movimentos de contracultura, quebra de padrões e revisão das relações estabelecidas um momento propício para uns dos momentos mais importantes da cultura DIY? Este artigo, através da breve contextualização deste período procura estabelecer um diálogo entre três literaturas escolhidas com base em revisão bibliográfica dos anos 1970 ao tratar do mobiliário nômade criado pela cultura Do-It-Yourself na década em questão: Nomadic Furniture volumes 1 e 2 dos autores James Hennessey e Victor Papanek e How to build your own living structures do autor Ken Isaacs.

Palavras Chave: DIY; década de 1970; mobiliário.

\begin{abstract}
It would be the 1970s with all its movements of counterculture, breaking patterns, and revision of the relations established a good time for some of the most important moments in DIY culture? This article, through the short contextualization of this period tries to establish a dialogue between three literatures chosen based on bibliographical review of the 1970s to deal with the nomadic furniture created by the Do-It-Yourself culture in the decade in question: Nomadic Furniture volumes 1 and 2 by the authors James Hennessey and Victor Papanek and How to build your own living structures by the author Ken Isaacs.
\end{abstract}

Keywords: DIY; 1970s; furniture. 


\section{Introdução}

A década de 1970 foi um período de extrema relevância para a cultura Do-It-Yourself - DIY devido suas condicionantes políticas e sociais, além de, uma série de "movimentos jovens de massa" que propiciaram o questionamento de uma série de padrões estabelecidos. Em 1971 o ativista social americano Jerry Rubin publica a obra We are everywhere, colocando-se contra o totalitarismo e a favor da liberação da mulher em relação aos homens e das utopias sobre novas formas de vida em comunidade.

Um grupo de fundamental importância para o aparecimento de tantos movimentos são os jovens Yippies durante o movimento de contracultura. Estes jovens foram responsáveis por difundir tais ideais pelo resto do mundo e era um grupo do qual pertenciam "mulheres, homens, gays" (RUBIN, 1971, p. 231, tradução nossa), que fizeram parte do Youth International Party.

Nesta época, Yippies militavam contra a Guerra do Vietnã (1955-1975), contra a discriminação racial da população negra, contra a ideia da organização tradicional familiar, contra a ideia da mulher ser tratada como uma "escrava do lar", da tecnologia quando utilizada como meio de oprimir a sociedade, contra a sociedade de consumo, contra o machismo, contra o capitalismo que divide o mundo em ricos e pobres (RUBIN, 1971).

Além de todas estas condicionantes existem fatores chaves para compreender as razões pelas quais esta década foi tão importante para a cultura do "Faça-Você-Mesmo" como, por exemplo, a crise do petróleo dos Estados Unidos que levou a uma situação de crise generalizada. É preciso compreender que a cultura DIY está ligada de maneira direta ao contexto econômico da qual pertence como, por exemplo, constatam Fineder; Geisler; Hackenschmidt (2016): em decorrência da ausência de profissionais especializados e como consequência da Segunda Guerra Mundial, com isso, há o crescimento de publicações e manuais que envolvem a temática do "FaçaVocê-Mesmo".

The Library of Radiant Optimism for Let's Re-make the World é uma biblioteca online criada pela iniciativa dos artistas Bonnie Fortune e Brett Bloom e como seus criadores e organizadores explicitam na homepage da biblioteca virtual houve o intuito de reunir os livros de contracultura importantes para as décadas de 1960 e 1970, pois, em suas concepções, embora nos dias de hoje existam problemas parecidos como os enfrentados nas décadas mencionadas, não existem movimentos de massa por parte das pessoas. Portanto, o intuito primordial dos autores da criação desta iniciativa é a difusão de livros que possam incentivar mudanças na atualidade.

A biblioteca conta com vinte e nove livros, sendo que a maioria deles está disponível para download em formato $p d f$ no site: < http://letsremake.info//rm/ > e tratam de temas como: práticas construtivas, contracultura, autoeducação, sociedade de consumo, desperdício, comunidade internacional, ativismo, portabilidade, design sustentável, DIY, pequenos espaços e estruturas nômades.

\section{Nomadic furniture 1 e 2}

Porém, dentre os livros da biblioteca, destacam-se três exemplares sobre mobiliário nômade voltados para a cultura DIY. Dois destes são os livros Nomadic furniture volumes 1 e 2 dos 

do autor Ken Isaacs ${ }^{3}$ chamado How to build your own living structures de 1974.

Figura 1 - Capa do livro Nomadic Furniture

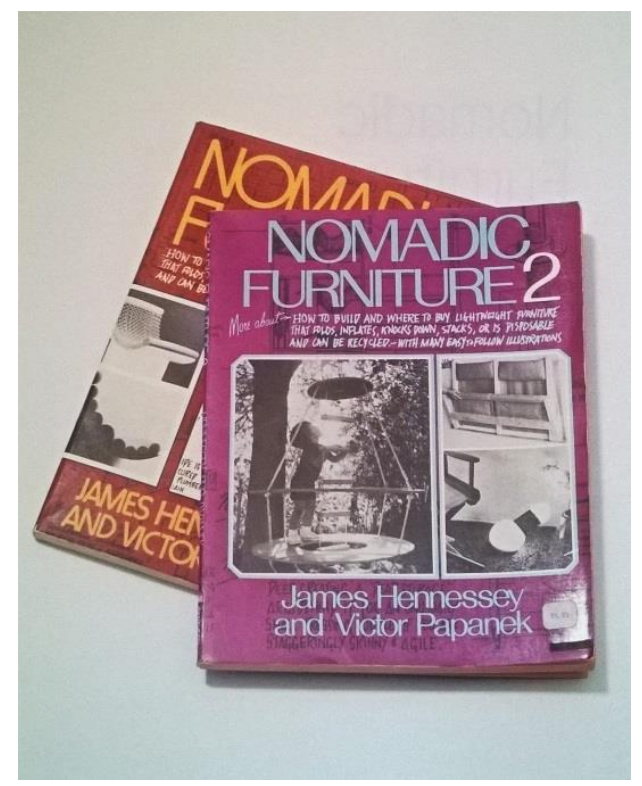

Fonte: Fineder, Geisler e Hackenschmidt (2016)

O primeiro paradigma quebrado com os livros Nomadic Furniture é que os autores não tiveram o intuito de produzir estes volumes para que apenas designers e arquitetos reproduzissem o seu conteúdo, como é comum neste tipo de publicação, mas quiseram dar a

\footnotetext{
${ }^{1}$ Segundo (PAPANEK; HENNESSEY, 1973, contracapa, tradução nossa): “James Hennessey é um designer industrial que obteve seu diploma de bacharel no Instituto de Tecnologia Illinois Institute of Design (...). Sua especialização é o projeto e a construção de dispositivos para cegos e deficientes e para pessoas do Terceiro Mundo".

2 Segundo as informações traduzidas da webpage < http://papanek.org/about/victor-j-papanek/ > da Papanek Foundation. “Victor J. Papanek (1923-1998), designer, professor e autor, nasceu em Viena, na Áustria, em 1923 (...). Educado na Cooper Union e no MIT, Papanek foi brevemente um aluno de Frank Lloyd Wright no início de sua carreira e tornou-se um seguidor e aliado de Buckminster Fuller que escreveu o prefácio da primeira edição em inglês da publicação seminal de Papanek Design for the Real World: Ecologia Humana e Mudança Social (1971). (...) As outras publicações de Papanek (coautoras com James Hennessey) incluem Nomadic Furniture I (1973), Nomadic Furniture II (1974); Como as coisas não funcionam (1977). Ele é o único autor de Design for Human Scale (1983) e The Green Imperative (1995). No decorrer de sua carreira, que durou até o final da década de 1990, Papanek aplicou os princípios do design socialmente responsável em projetos colaborativos com preocupações como a UNESCO e a Organização Mundial de Saúde".

${ }^{3}$ Ken Isaacs nasceu em Peoria, nos Estados Unidos em 1927, estudou na Bradley University e, por meio de uma bolsa de estudos da Cranbrook Academy of Art, fez mestrado entre 1952 e 1954 nessa instituição. Em 1956 é chamado para assumir o cargo de chefe do Departamento de Design da Cranbrook, permanecendo até 1957. Ainda como docente, lecionou por breves períodos na Escola de Design de Rodhe Island, no Instituto de Design do Illinois Institute of Technology e por fim na Faculdade de Arquitetura da Illinois University em Chicago. Atuou em alguns escritórios, teve o seu em Nova York e a sua própria fábrica de móveis. Ficou conhecido pelo seu trabalho com matrizes, a Estrutura Viva e as Microcasas. Em 1968 foi convidado para ser consultor da revista de DIY, Popular Science, publicando vários projetos. Em 1974 publicou o livro: How to build your own living structures.
} 
oportunidade para que pessoas comuns, sem formação na área, apropriem-se destes conteúdos para que pudessem criar seus próprios móveis, por meio de um design simples, fácil de montar e de transportar e, principalmente, adequado às necessidades reais da realidade cotidiana de seus leitores.

Figura 2 - O livro é o resultado da aplicação do seu conteúdo no cotidiano dos autores

Figura 3 - Exemplo de soluções projetuais com detalhes construtivos
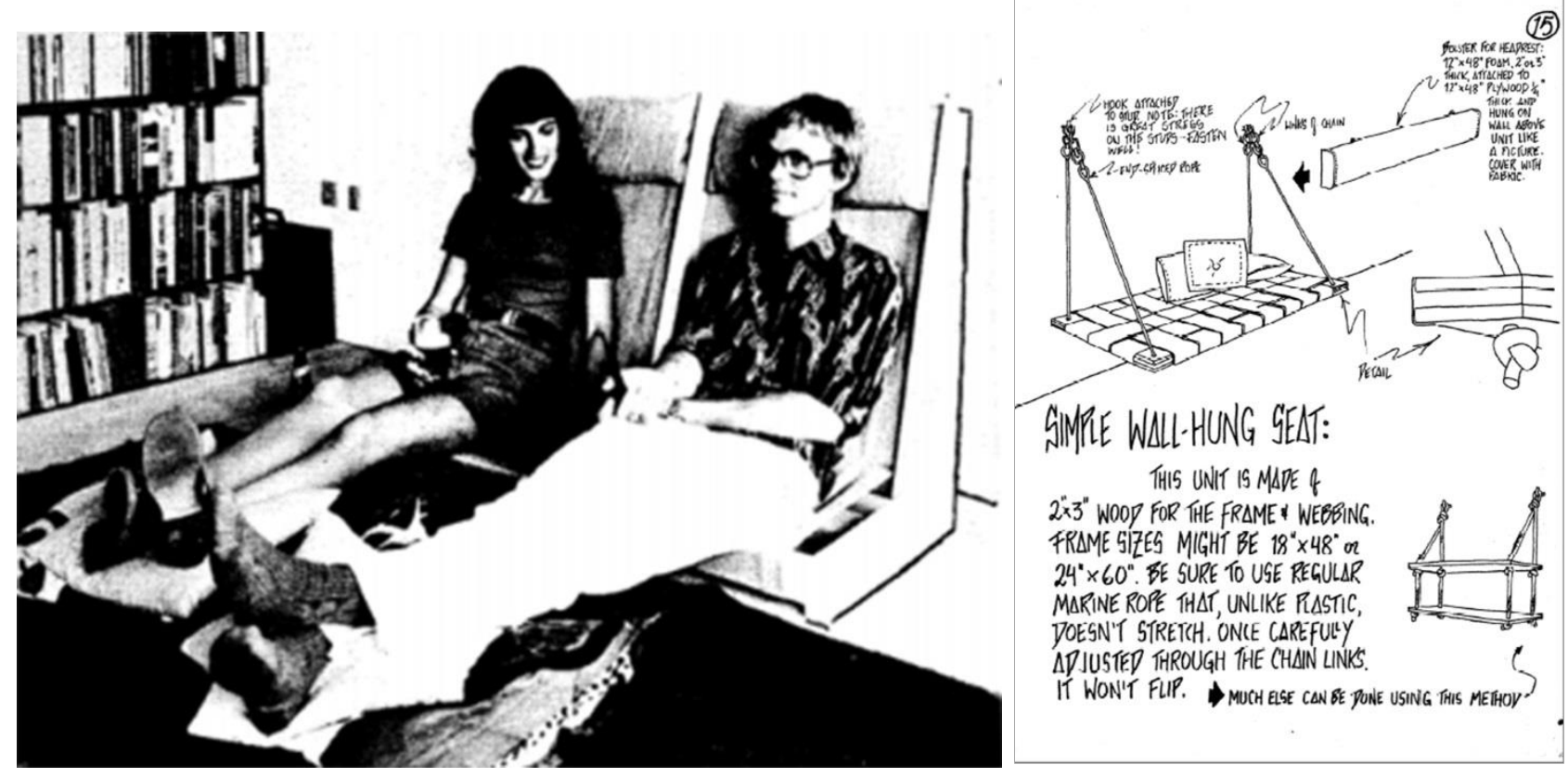

Fonte: Papanek e Hennessey (1974)

É importante salientar que os autores tiveram um estilo de vida que se aproximava das condições de nomadismo contemporâneo, sendo este compreendido pelas autoras deste artigo como sendo uma vida de constantes deslocamentos territoriais, além disso, são favoráveis ao acesso das pessoas ao design e a desmistificação deste campo do conhecimento, ao o tornar acessível para todos. Assim, este tipo específico de mobiliário não convencional ganhou destaque. Como destaca James Hennessey: "A primeira edição do livro vendeu mais de 10.000 cópias e rapidamente se tornou um best-seller (...)" em (FINEDER; GEISLER; HACKENSCHMIDT, 2016, p. 8, tradução nossa ${ }^{4}$ ). Aliás, os autores Papanek e Hennessey ressaltam que as publicações de design negligenciaram os móveis desmontáveis e compactáveis ou, o que eles chamam de móveis nômades e o sucesso do primeiro livro foi tamanho que o segundo volume foi escrito também com sugestões enviadas pelos leitores do primeiro. Todo este contexto propiciado pela década de 1970 favoreceu o aparecimento de móveis não convencionais.

O design de luxo também é criticado, visto que, com tantos os problemas evidentes e movimentos de lutas sociais existentes no período como foi destacado na introdução deste estudo, os autores questionam a irrelevância de se pensar um design não acessível, além disso, acreditam que não adianta buscar refúgio da realidade em artefatos, desta forma, são priorizadas soluções econômicas que, ao mesmo tempo, tenham bom funcionamento.

\footnotetext{
${ }^{4}$ Texto original: The first edition of the book sold over 10.000 copies and quickly became a bestseller (...).
} 
Figura 4 - Página do livro Nomadic Furniture

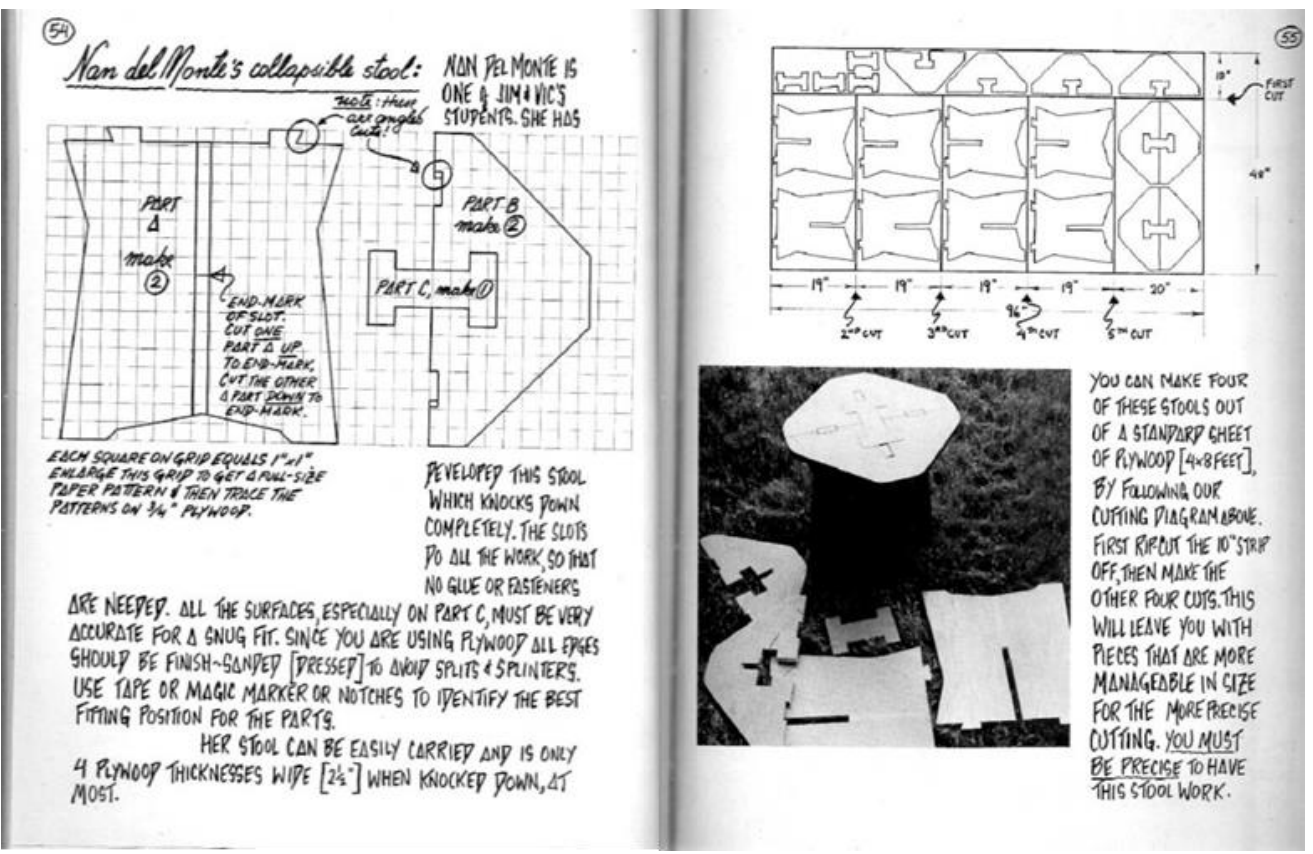

Fonte: Papanek e Hennessey (1973)

Outra questão importante a ser destacada é que estes livros são pioneiros ao destacarem aspectos ambientais no design e, surpreendentemente, demonstram uma visão mais ampla sobre este aspecto ao mencionarem que: "O verdadeiro valor deste livro é que ele irá permitir todos nós nômades ter mais possuindo menos" (PAPANEK; HENNESSEY, 1974, p. 3, tradução nossa) $)^{5}$. Além de ser uma crítica evidente à sociedade de consumo da época, o que se pode deduzir da abordagem proposta por James Hennessey e Victor Papanek é que os autores incentivam uma vida com menos bens materiais para se ter uma vida com mais liberdade e desprendimento, para poder viajar mais, visto que, como foi apresentado no início, uma das proposições da época é o questionamento sobre a necessidade da existência de fronteiras entre os povos.

Os livros se constituem através de dicas, desenhos, detalhamentos e fotografias destes móveis e organizam o conteúdo fornecido por meio das categorias como: sentar, comer e trabalhar, armazenar, dormir, iluminar, dicas de trabalho. Além de estudos sobre as medidas humanas e sugestões para auxiliar os seus leitores a construírem estes móveis como o uso de ferramentas e dos materiais necessários. É importante salientar que o segundo volume, corresponde a um livro novo e não apenas uma atualização do primeiro.

\footnotetext{
${ }^{5}$ Texto original: The real value of this book is that it will enable all of us nomads to have more by owning less.
} 
Figura 5 - Habitácolo projetado por Bruno Munari

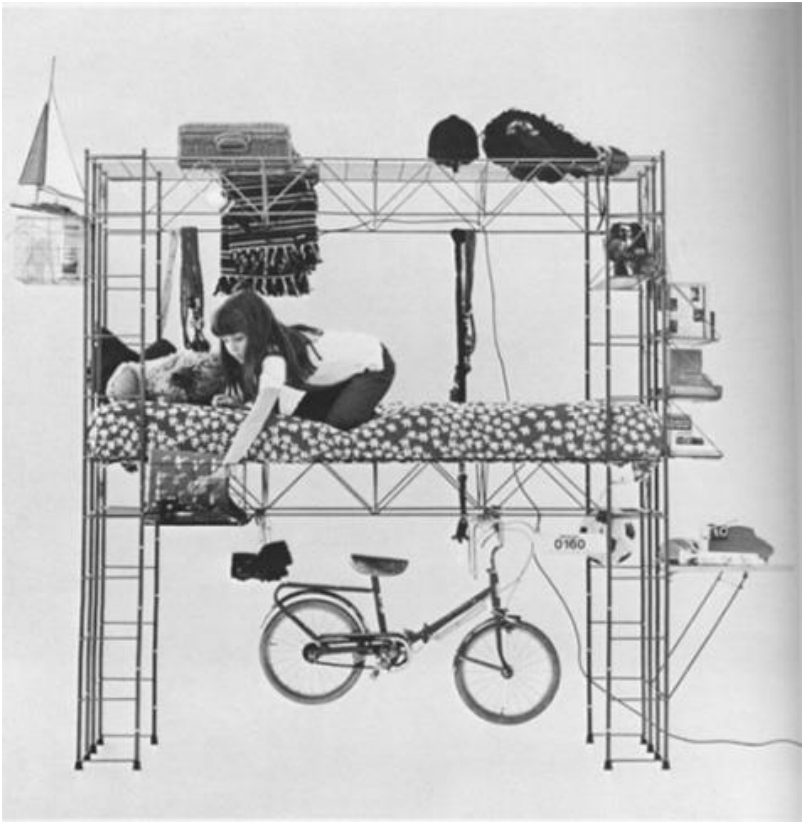

Fonte: Multiplode6.com (1971)

Bruno Munari desenvolve o Habitácolo, um móvel que gera inúmeras possibilidades de apropriação. Segundo texto do autor: “(...) Habitáculo é o espaço habitável na medida do essencial. Em sentido figurado é também o íntimo recanto individual, o lugar onde se situa tudo aquilo que forma o mundo de alguém. (...) É o mínimo que oferece o máximo. (...)" (MUNARI, 1998, p. 190 e 191). E o livro Nomadic furniture nos apresenta o Relaxation Cube e o Work Cube abaixo, bem como o Entertaining Cube e o Children's Cube.

Figura 6 - Relaxation Cube e Work Cube

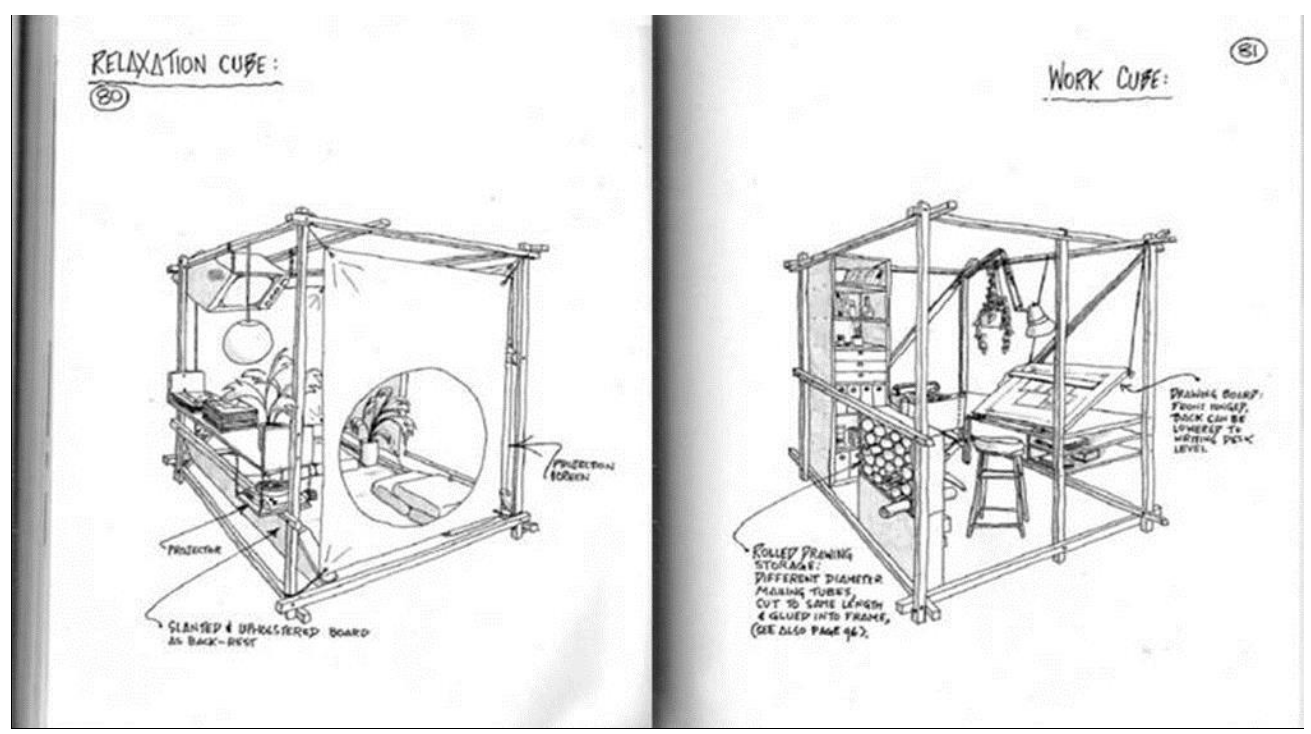

Fonte: Papanek e Hennessey (1973) 


\section{How To Build Your Own Living Structures}

Figura 7 - Capa do livro How To Build Your Own Living Structures

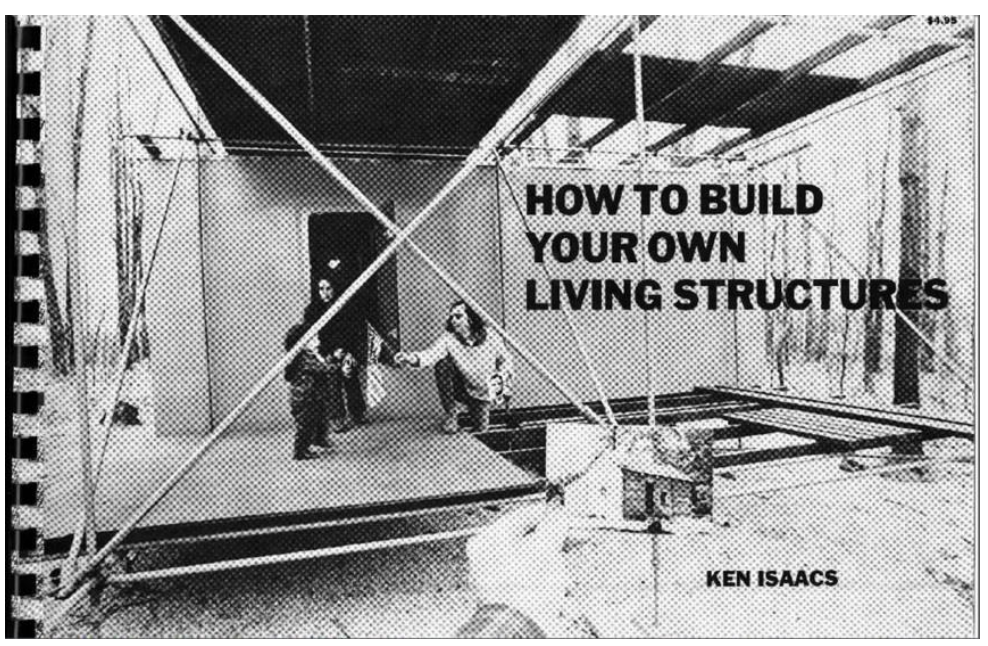

Fonte: Isaacs (1974)

Ken Isaacs publicou no ano de 1974 o livro How To Build Your Own Living Structures e, além de ser um guia DIY, ele merece destaque dentre tantos guias que contribuíram para a cultura de desenvolvimento de objetos na década de 1970, ao acrescentar sugestões que envolvem tanto a arquitetura quanto o design de espaços nada convencionais inclusive para os dias de hoje. Este desenvolvimento se deve em grande parte a ideia de matriz, sendo este um ponto fundamental para a proposição do espaço por ele ${ }^{6}$. Na figura abaixo, há a manifestação da preocupação em liberar o espaço do ambiente.

Figura 8 - Espaço livre para circulação baseado em móveis flexíveis

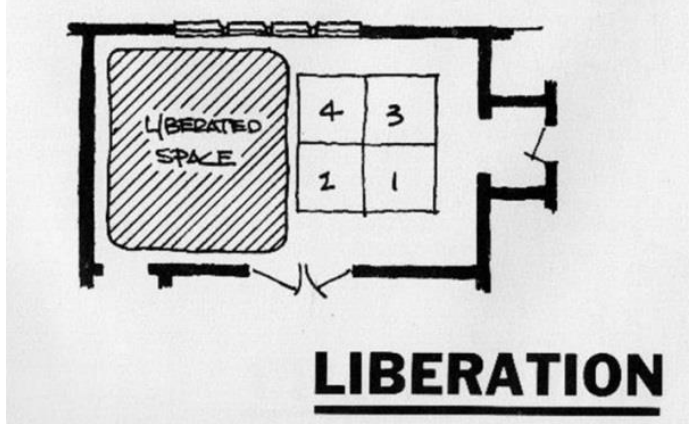

Fonte: Isaacs (1974)

A atenção especial dada aos móveis justifica-se pelo fato de que Ken Isaacs considerou o fato de que: "Espaço liberado/ móveis tradicionais nunca foram organizados como um sistema inteiro" (ISAACS, p. 35, 1974, tradução nossa ${ }^{7}$ ). E ainda: “Esforços fracos foram feitos para

\footnotetext{
${ }^{6}$ Sobre a trajetória profissional de Isaacs e o desenvolvimento contínuo e único da ideia de matriz, no ensino e na prática, consultar artigo de Margolin (2014).

${ }^{7}$ Texto original: Liberated space/ traditional furniture was never organized as a whole system.
} 
organizá-los "visualmente", mas isso foi apenas outra armadilha" (ISAACS, p. 35, 1974, tradução nossa ${ }^{8}$.

Portanto, seus móveis estavam alinhados com o conceito de grid. Visto que, segundo as palavras do próprio autor: "Eu chamo o grid de 'consistente' porque ele pode ser preenchido em uma ou todas as três direções principais no espaço sem problemas ou comprometimento (ou condições especiais não resolvidas) apenas adicionando o tamanho único (...)" (ISAACS, 1974, p. 57 , tradução nossa ${ }^{9}$ ).

Figura $9-\ln$

Figura 10-Out

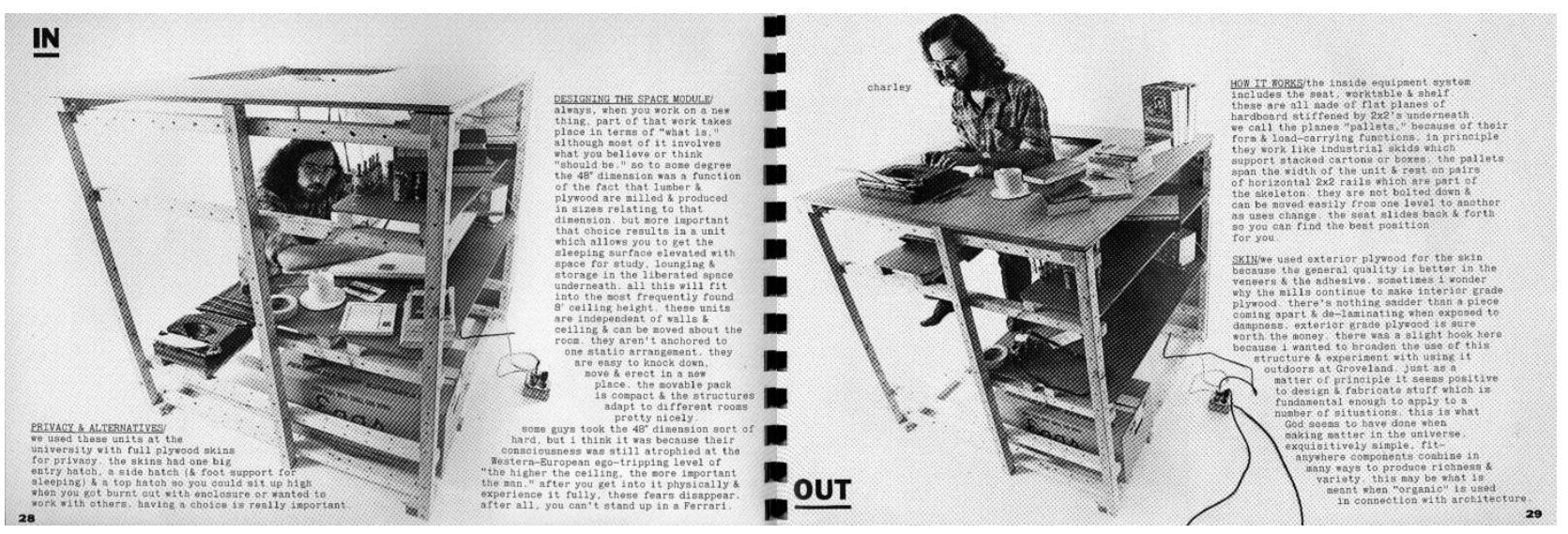

Fonte: Isaacs (1974)

Figura 11 - Super Chair

Figura 12 - Super Chair: usos inclusos em um só móvel

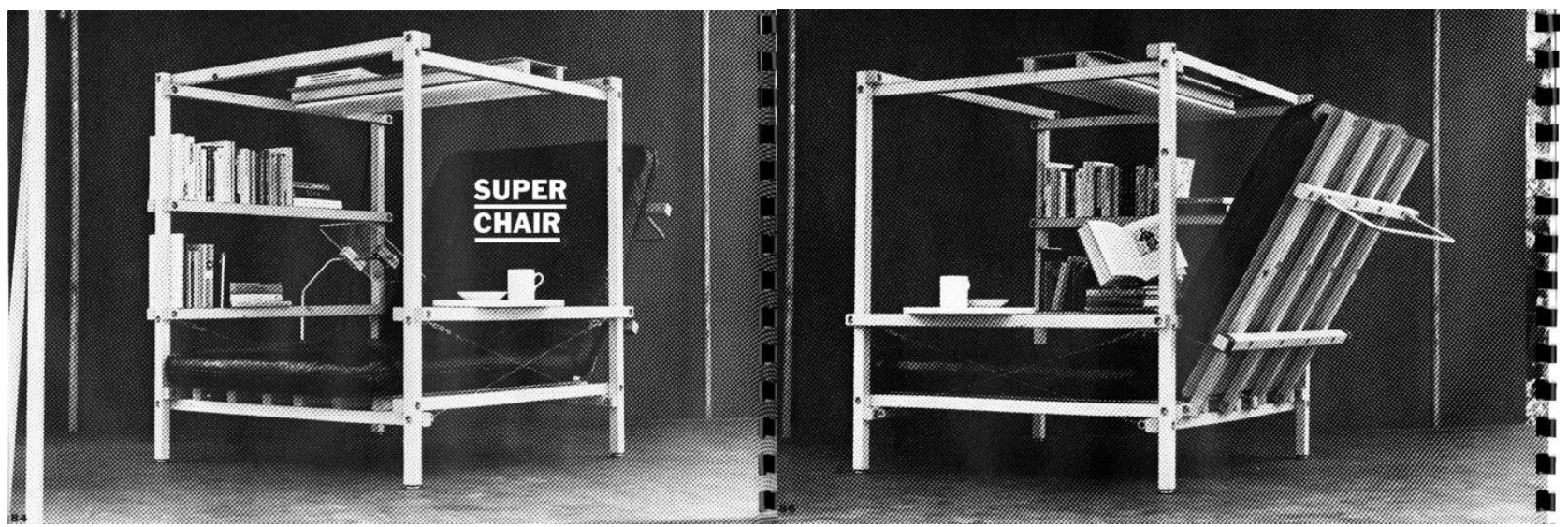

Fonte: Isaacs (1974)

Outra preocupação do designer além do produto em si que poderia ser obtido era referente a uma visão consciente do processo mental que abrange as atividades que se relacionam com a produção de artefatos. Esta crítica se passa em um tempo em que as mídias da

\footnotetext{
${ }^{8}$ Texto original: Feeble efforts were made to organize them "visually" but that was just another trap.

9 Texto original: I call the grid "consistent" because it can be filled in any or all of the three main directions in space without problems or compromise (or special unresolved conditions) just by adding the one-size (...).
} 
época eram em sua maioria analógicas, mas Ken Isaacs já observava que facilidades e aparatos tecnológicos poderiam contribuir para alterações de relações naturais do homem em suas atividades cotidianas:

A magia: a limitação das condicionantes culturais que é a marca dos supervendedores da mídia tem sutilmente prejudicado nossa capacidade de concentração e deformado severamente nossa relação com o tempo. Misturando cartões de crédito e assinando contratos de pagamento mensais que diminuíram a nossa capacidade de lidar com as atividades em tempo real de fazer e ser (ISAACS, 1974, p. 4, tradução nossa ${ }^{10}$ ).

Não poderia ser diferente sua relação com o meio ambiente, este é visto pelo autor como um elemento capaz de libertar as pessoas de se tornarem distanciadas das suas relações com a Terra devido às tecnologias.

Vi e senti a necessidade de grandes simplificações e o reconhecimento de relações positivas com a Terra e terapia de mudança ambiental para liberar todos nós dos maníacos das altas tecnologias (ISAACS, 1974, p. 3, tradução nossa ${ }^{11}$ ).

A publicação traz uma mensagem de simplicidade e de emancipação de bens pessoais para a valorização da liberdade assim como a ideia norteadora dos livros Nomadic Furniture. Além disto, as propostas dos livros instigam com que seus leitores intensifiquem as transmissões de conhecimento entre as pessoas a partir das relações desenvolvidas com as proposições de projeto, o que também se traduz no espírito DIY.

Outro reflexo do pensamento vigente da época são as quebras de padrões sobre as estruturas patriarcais e a divisão convencional do trabalho entre homens e mulheres. Na imagem abaixo, como observam Fineder; Geisler; Hackenschmidt (2016), Ken e Carole Isaacs subvertem os papéis atribuídos da maneira tradicional a cada membro. E, com isto, ilustram a importância que a arquitetura e o design devem assumir como meios e agentes de reflexão nos períodos dos quais emergem.

\footnotetext{
${ }^{10}$ Texto original: The magic/ the culture-conditioning limitation that is the imprint of the media supersalesmen has slightly wrecked our ability to concentrate e severely warped our time-sense. Shuffling credit cards and signing monthly payment agreements have decresed our ability to handle the real-time activities of making and being.

${ }^{11}$ Texto original: I saw and felt the necessity for major simplifications, and recognition of positive earth relationships and environmental change-therapy to release us all from the high-techs maniacs.
} 
Figura 13 - Quebra de padrões em estruturas familiares

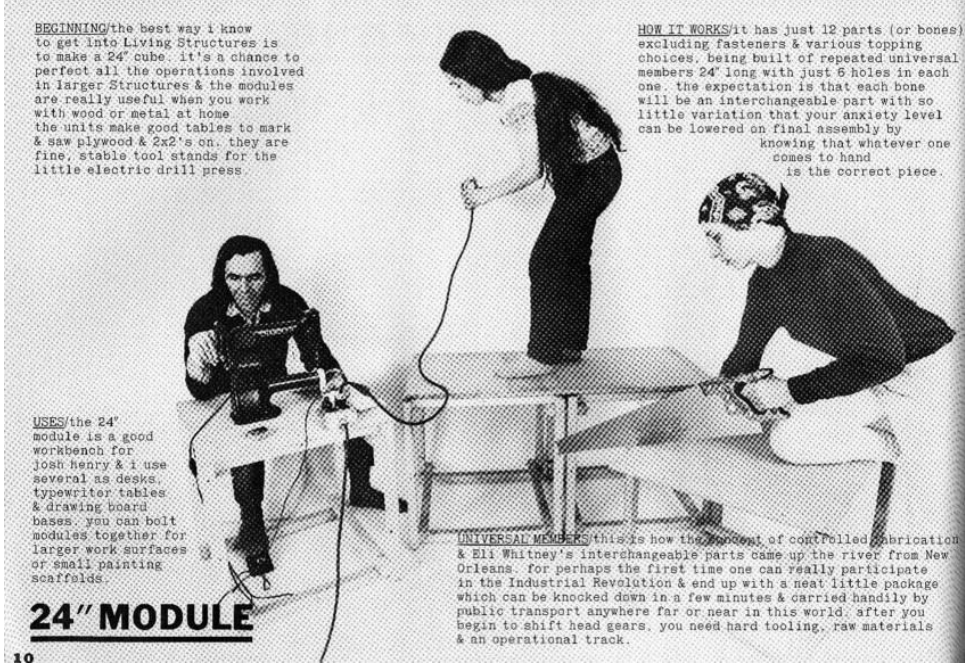

Fonte: Isaacs (1974)

\section{Considerações finais}

A partir de tais reflexões, pode-se inferir que o movimento DIY encontra na década de 1970 as condições adequadas para sua manifestação. Os exemplos aqui apresentados possuem alta relevância na contemporaneidade porque, a cultura "Faça-Você-Mesmo" se encontra novamente em evidência devido aos ambientes de fab labs e das comunidades de frequentadores destas redes que operam com o compartilhamento de seus projetos em bibliotecas virtuais como uma nova realidade de aplicação do DIY.

Como pode ser observado na animação "Why" disponível no site < https://www.ponoko.com/ > existem comunidades virtuais de compartilhamento de projetos para serem feitos por fabricação digital, nelas pessoas em qualquer lugar do mundo podem baixar arquivos para serem materializados em qualquer fab lab, além disso, os projetos podem ser customizados e podem dar a possibilidade de personalização. O que novamente convida as pessoas a cocriarem.

Por outro lado, como hoje estes processos ocorrem em sua maioria na rede e são materializados por meios digitais, as práticas de "Faça-Você-Mesmo" são cada vez menos manuais, o que não significa que se tornaram alienadas, visto que, este processo apesar de ser digital só é possível de ser realizado com a interferência e participação humana em todas as etapas.

Outro ponto relevante a ser destacado é que os autores dos três livros têm em comum uma preocupação constante em se aproximar de seu público com uma linguagem simples e objetiva, sendo pioneiros ao tratar de questões ambientais no design com uma mensagem de claro incentivo ao desapego de bens em plena sociedade de consumo. Hoje, mais uma vez, estas questões se colocam, e saber quando não produzir objetos e saber quando não os consumir devem ser discutidos pelos seus usuários/ produtores.

Trata-se, portanto, de três obras da década de 1970, com uma proposta ainda compatível com a atualidade que vive outro momento da cultura do "Faça-Você-Mesmo". Assim, espera-se 
que este artigo possa ajudar a estabelecer relações com o momento presente com o mesmo espírito propositivo e reflexivo de outrora.

\section{Referências}

FAB LAB LIVRE SP. 2017. Disponível em: < http://www.fablablivresp.com.br/ >. Acesso em: 17 mar. 2017.

FINEDER, M.; GEISLER, T.; HACKENSCHMIDT, S. Nomadic Furniture 3.0: new liberated living?. Viena: Mak Studies, 2016.

ISAACS, K. How To Build Your Own Living Structures. New York: Harmony Books, 1974.

LET'S RE-MAKE. Disponível em: < http://letsremake.info/Irm/ >. Acesso em: 25 dez. 2017.

MARGOLIN, V. Ken Isaacs: designer de matrizes. In: Políticas do artificial: ensaios e estudos sobre design. Rio de Janeiro: Record, 2014, p. 81-103.

MULTIPLODE6. Disponível em: < www.multiplode6.com >. Acesso em: 02 abr. 2018.

MUNARI, B. Das coisas nascem coisas. São Paulo: Martins Fontes, 1998.

PAPANEK FOUNDATION. Disponível em: < http://papanek.org >. Acesso em: 17 mar. 2017.

PAPANEK, V.; HENNESSEY, J. Nomadic Furniture. New York, Toronto: Pantheon Books, 1973.

PAPANEK, V.; HENNESSEY, J. Nomadic Furniture 2. New York, Toronto: Pantheon Books, 1974.

PONOKO. Disponível em: < https://www.ponoko.com/ >. Acesso em: 03 abr. 2018.

RUBIN, J. We Are Everywhere. Harper Colophon Books, 1971. 